Vibrational cooling of spin-stretched dimer states by He buffer gas: Quantum calculations for $\operatorname{Li}_{2}\left(a^{3} \Sigma_{u}^{+}\right)$at ultralow energies

S. Bovino, E. Bodo, E. Yurtsever, and F. A. Gianturco'

Citation: The Journal of Chemical Physics 128, 224312 (2008); doi: 10.1063/1.2933405

View online: http://dx.doi.org/10.1063/1.2933405

View Table of Contents: http://aip.scitation.org/toc/jcp/128/22

Published by the American Institute of Physics

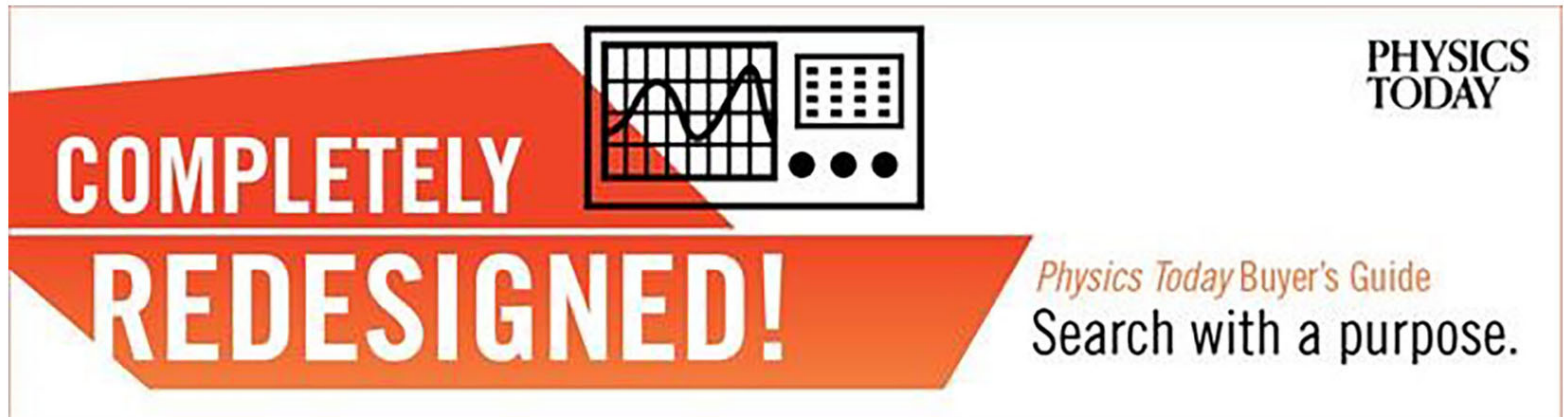




\title{
Vibrational cooling of spin-stretched dimer states by He buffer gas: Quantum calculations for $\operatorname{Li}_{2}\left(a^{3} \Sigma_{u}^{+}\right)$at ultralow energies
}

\author{
S. Bovino, ${ }^{1}$ E. Bodo, ${ }^{1}$ E. Yurtsever, ${ }^{2}$ and F. A. Gianturco ${ }^{1, a)}$ \\ ${ }^{1}$ Department of Chemistry and CNISM, University of Rome La Sapienza, Piazzale Aldo Moro 5, \\ 00185 Rome, Italy \\ ${ }^{2}$ Department of Chemistry, Koç University, Rumelifeneriyolu, 34450 Sariyer, Istanbul, Turkey
}

(Received 24 January 2008; accepted 30 April 2008; published online 12 June 2008)

\begin{abstract}
The interaction between the triplet state of the lithium dimer, ${ }^{7} \mathrm{Li}_{2}$, with ${ }^{4} \mathrm{He}$ is obtained from accurate $a b$ initio calculations where the vibrational dependence of the potential is newly computed. Vibrational quenching dynamics within a coupled-channel quantum treatment is carried out at ultralow energies, and large differences in efficiency as a function of the initial vibrational state of the targets are found as one compares the triplet results with those of the singlet state of the same target. () 2008 American Institute of Physics. [DOI: 10.1063/1.2933405]
\end{abstract}

\section{INTRODUCTION}

In the past few years, our capabilities of controlling the behavior of ultracold atomic gases have had notable successes, such as the controlled implosion of Bose-Einstein condensates ${ }^{1}$ and the production of molecules within both bosonic $^{2,3}$ and fermionic ${ }^{4,5}$ quantum gases. Such improved capabilities have also had important effects in other areas, such as the analysis of the transition between Bose-Einstein condensation and Bardeen-Cooper-Schrieffer behavior in dilute gases. ${ }^{6,7}$

In parallel, there have also been intense efforts to cool molecular systems directly from the higher temperatures to the ultracold regime. For example, $\mathrm{NH}_{3}, \mathrm{OH}$, and $\mathrm{NH}$ have been cooled down to the millikelvin regimes by using buffergas cooling. ${ }^{4,5,8}$ Such directly cooled molecular systems have been successfully trapped around $10 \mathrm{mK}$, and several proposals exist for cooling them further down via evaporative and sympathetic cooling methods. ${ }^{8}$ Because of the greater complexity present in molecular systems and the more complicated interplay of their internal degrees of freedom during the quenching process, ${ }^{9,10}$ the possibility of controlling the molecular interactions and the initial molecular conditions in the same way as in atomic interactions ${ }^{11}$ has naturally attracted a great deal of interest, both experimentally and theoretically, ${ }^{12}$ since the changes in the behavior of the relevant scattering attributes at ultralow energies have been found to be strongly affected by controlled changes of the interactions at play. More specifically, we have recently shown from accurate computations that the efficiency of relaxing an internally "hot" molecular partner in ultracold traps depends markedly on the initial state of the molecule because of the effects that the selected initial conditions have on the de-excitation cross sections. ${ }^{13,14}$

In general, it may be possible to produce beams of cold and ultracold molecules, which can be used as a "probe" against various buffers for molecular scattering properties

\footnotetext{
${ }^{a)}$ Author to whom correspondence should be addressed. Electronic mail: fa.gianturco@caspur.it. FAX: +39.06.49913305.
}

near the threshold. The buffers can be made of neutral molecules or atoms, as in the experiments reported in Ref. 15 where a rare gas atom was used, or of an array of molecular and atomic ions such as those contained in a Coulomb crystal. ${ }^{16}$ Among the various sources of ultracold molecules, the photoassociation has been succesfully employed to obtain triplet diatoms of alkali metals. Therefore, in the present work, we shall examine the behavior of neutral $\mathrm{Li}_{2}$ interacting with ${ }^{4} \mathrm{He}$ and consider the changes induced by varying two physical properties of that molecular partner: its spin state, since we consider the molecule being formed in its triplet state ${ }^{3} \Sigma_{u}^{+}$, and its vibrational state, since we consider all the different vibrational levels of $\mathrm{Li}_{2}$ as possible initial states for the collision.

Although it is still difficult to predict when experimental data on this system could become available at the ultralow energies in the range we have studied here, we think that to have accurate calculations available as a comparison with those we already have for the singlet case constitutes in itself a task worth pursuing because of what one can learn from it as a general problem.

The calculations have been carried out to cover very low temperatures, from millikelvins to microkelvins, and convergence of the results has been ensured over the whole range although experiments down to microkelvins are still to be achieved.

The work is organized as follows: Section II describes the $\operatorname{Li}_{2}\left({ }^{3} \Sigma_{u}^{+}\right)$interaction with ${ }^{4} \mathrm{He}$ atoms as a function of the internal Jacobi coordinates and the fitting procedure employed for the initial raw points, which were obtained from the ab initio calculations. Section III reports the quantum inelastic scattering processes as a function of the initial rotovibrational level of the triplet target and details the coupled-channel results from our calculations. Section IV summarizes our present conclusions.

\section{THE TRIPLET POTENTIAL ENERGY SURFACE}

Calculations involving the rigid-rotor interaction of the triplet state of $\mathrm{Li}_{2}$, the $a^{3} \Sigma_{u}^{+}$state, with He have already been 

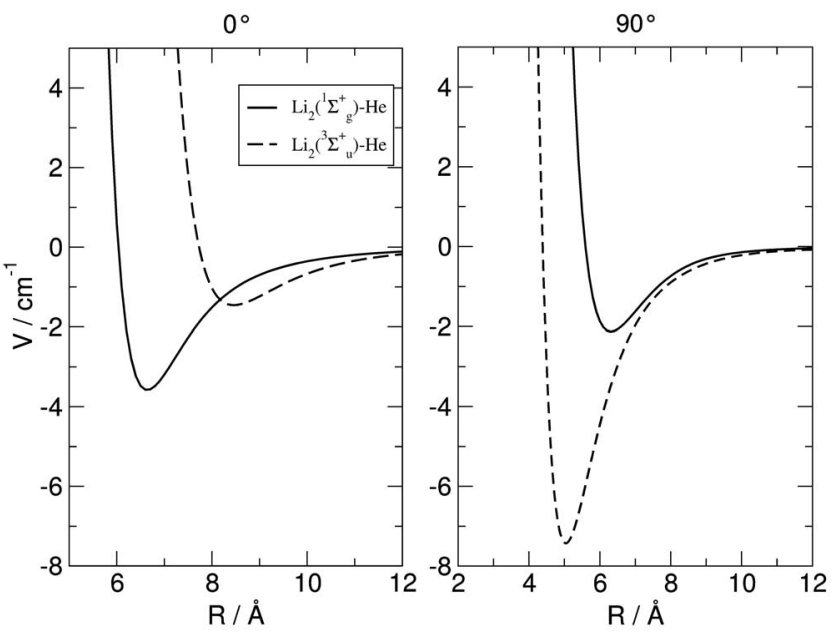

FIG. 1. Computed PESs for the interaction of $\operatorname{Li}_{2}\left(a^{3} \Sigma_{n}^{+}\right)-\mathrm{He}$ (dashes) and of $\mathrm{Li}_{2}\left({ }^{1} \Sigma_{\mathrm{q}}^{+}\right)-\mathrm{He}$ (solid lines) at two different angular orientations: $\vartheta=0^{\circ}$ (left panel) and $\vartheta=90^{\circ}$ (right panel). The data for the singlet state are from Ref. 17.

presented by us before and have been described in detail in Ref. 17. We have further tested the potential energy surface (PES) by evaluating the rotationally inelastic scattering attributes in the low- $T$ range up to $100 \mathrm{~K}$ and compared their values with those obtained from the ionic dimer species, $\mathrm{Li}_{2}^{+}\left(X^{1} \Sigma_{g}^{+}\right) .{ }^{18}$

The structural changes that occur on the interaction of the molecular partner with $\mathrm{He}$ when going from the dimer singlet state to the triplet state could be appreciated by the pictorial comparison organized in the two panels of Fig. 1 (see also Ref. 19).

One clearly sees in that figure the marked orientational differences between the two rigid-rotor PESs: the singlet target (solid lines) and the triplet one (dashes). The T-shaped approach, in fact, is seen to produce a deeper attractive well for the triplet case and a more compact configuration with the onset of the repulsive wall occurring around $4 \AA$. On the other hand, for the case of the singlet target, it is the collinear configuration which exhibits a deeper well and also shows for that cut of the PES a less compact complex configuration: a repulsive wall with its onset around $6 \AA$.

The rigid-rotor $a b$ initio energies have been obtained using the MP4 method with the cc-pV5Z basis set and keeping the $r_{\mathrm{eq}}$ value for the triplet partner at $4.175 \AA \AA^{17}$ The interaction potential has therefore been extended in the present work by generating a new set of intramolecular geometries over a range of intermolecular $R$ values from 3.5 to $14 \AA$ for a total of 4726 points. The Jacobi angles used were in the range of $\theta=0^{\circ}-90^{\circ}$. Hence, once we add the previous rigidrotor geometries, we finally have a total of 5638 points. The resulting PES is a function of the two previous Jacobi coordinates plus the orientation angle $\vartheta$,

$$
V(R, r, \theta)=E(R, r, \vartheta)-E_{\mathrm{Li}_{2}}(r)
$$

It was then analytically represented by first generating the nonseparable three-body interaction,

$$
V_{3 B}=V(R, r, \theta)-V\left(r_{\mathrm{Li}_{A}-\mathrm{He}}\right)-V\left(r_{\mathrm{Li}_{B}-\mathrm{He}}\right),
$$

which was fitted, in turn, by using an Aguado-Paniagua type of expansion ${ }^{20}$ as implemented in our group: The final standard deviation was $\sigma=0.047 \mathrm{~cm}^{-1}$. The total number of points was also reduced by excluding the repulsive energy values above $5000 \mathrm{~cm}^{-1}$ with the use of a simple weight function: Our final interaction potential was then constructed by summing the three-body contribution of Eq. (2) to the two accurate empirical $\mathrm{Li}-\mathrm{He}$ diatomic potentials that we have taken from Ref. 21.

Although it is difficult to properly assess the quality of the computed PES since we do not have experimental observables to test our calculations with, within the realm of what we consider a comparative assessment between similar but different systems like the triplet and singlet states of the lithium dimer, we think that the present results would not change much when improved PES were to be used. One should also note that our calculations ${ }^{19}$ employ an empirical adjustment of the interaction that correctly reproduces the best atom-atom potential curve available for this system. ${ }^{21}$

The structural differences between the spin-stretched state of the dimer and the singlet could be further appreciated by comparing the vibrational sequence of bound states existing in the ${ }^{3} \Sigma_{u}^{+}$with those for the ${ }^{1} \Sigma_{g}^{+}$. Recent calculations of the vibrational levels ${ }^{22}$ found the same numbers that we obtained and indicated the best dissociation energy $D_{e}$ to be around $334.145 \mathrm{~cm}^{-1}$, with a $D_{0}$ value of $301.989 \mathrm{~cm}^{-1}$. Our present calculation employs very similar values: $D_{e}$ $=335 \mathrm{~cm}^{-1}$ and $D_{0}=302 \mathrm{~cm}^{-1}$. The spin-stretched state is able to support only about ten vibrational levels. For comparison the singlet has a $D_{e}$ of $8516.78 \mathrm{~cm}^{-1}$ and supports 42 vibrational states. ${ }^{23}$ The rotational constants $B_{\text {rot }}$ are also different since we have $B_{\text {rot }}^{\text {triplet }}=0.26681 \mathrm{~cm}^{-1}$ and $B_{\text {rot }}^{\text {singlet }}$ $=0.67264 \mathrm{~cm}^{-1}$. Thus, as much as possible, we verify the overall reliability of the present PESs with the above set of comparisons.

Another interesting indicator of the structural differences between the two states could be seen by looking at a comparison between the coefficients of the multipolar expansion of the interactions, which couples the various vibrational levels of the triplet target,

$$
\begin{aligned}
V_{v v^{\prime}}(R, \vartheta) & =\int d r \varphi_{v}^{*}(r) V(R, \vartheta, r) \varphi_{v^{\prime}}(r) \\
& =\sum_{\lambda=0}^{\lambda_{\max }} V_{v v^{\prime}}^{\lambda}(R) P_{\lambda}(\cos \vartheta)
\end{aligned}
$$

The four panels in Fig. 2 report the changes in the interaction region around the strongest potential well (for the T-shaped geometry) occurring for four different intermolecular distances of the vibrating triplet target. We observe in Fig. 2 that the projectile penetrates more closely into the molecule, as the bond stretches across the range of values sampled by the vibrational states, than was the case for the singlet state of $\mathrm{Li}_{2}{ }^{24}$

Figure 3 compares the features of the off-diagonal terms of the vibrational coupling potentials [those of Eq. (3)] computed at the two orientations of $\mathrm{He}-\mathrm{Li}_{2}$, which provide the 

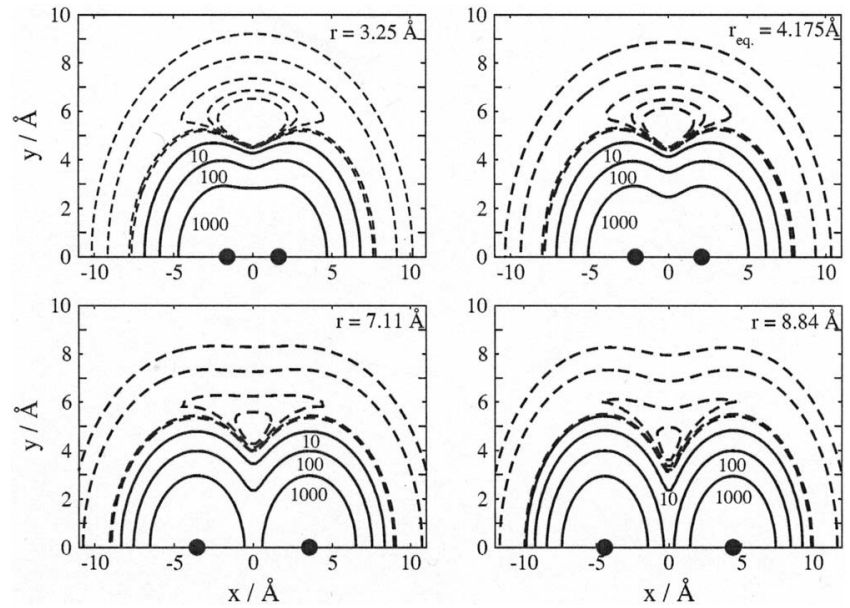

FIG. 2. Computed isoenergy lines for the PES of the triplet target as a function of the intramolecular bond distance. The energy units are in $\mathrm{cm}^{-1}$; distances are $\AA$.

strongest interactions: at $\vartheta=90^{\circ}$ for the triplet target and at $\vartheta=0^{\circ}$ for the singlet one. The relevant vibrational wavefunctions have been obtained via the $\mathrm{Li}_{2}$ triplet potential given by the numerical analysis of Refs. 23 and 25. One sees that ${ }^{3} \Sigma_{u}^{+}$ presents potential slightly stronger radial couplings between channels, which become strongly attractive at short range, while the singlet case for highly excited vibrational states exhibits a repulsive short-range behavior, which excludes encounters at close quarters that would enhance the vibrational couplings. This specific effect will be shown to play an important role when discussing the quenching efficiency in the next section.

Another additional parameter of the interaction, which is interesting to compare for the two systems, is the orientational anisotropy of the lowest diagonal terms of the vibrational coupling matrix (i.e., the one corresponding to $\nu$ $\left.=0, \nu^{\prime}=0\right)$. In particular, we wish to compare the behavior between coefficients of the multipolar expansion from Eq. (3): Those terms, in fact, are directly responsible for the transitions between rotational levels separated by different $\Delta j$
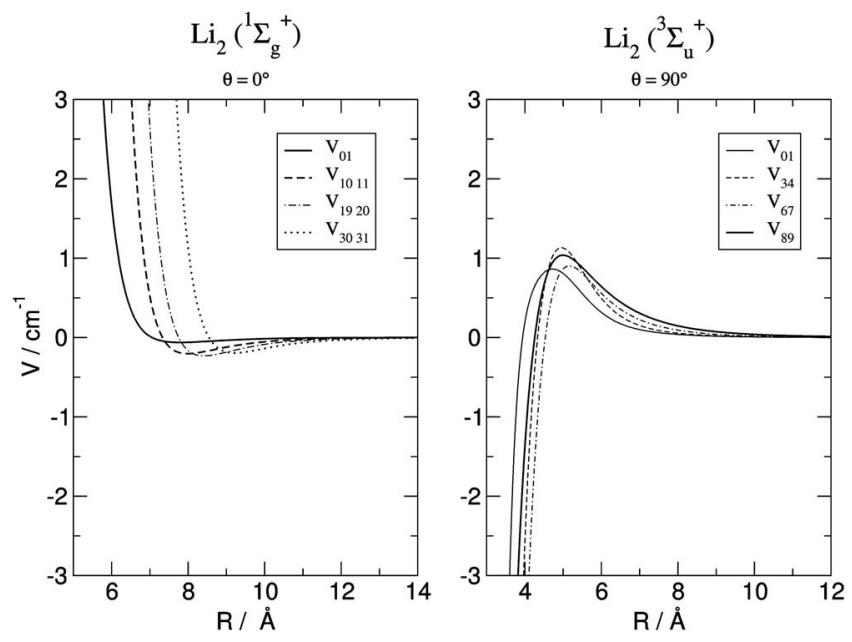

FIG. 3. Computed vibrational coupling potential terms (off-diagonal) at the relative orientations corresponding to the strongest interactions for the singlet (left) and triplet (right) molecular targets.
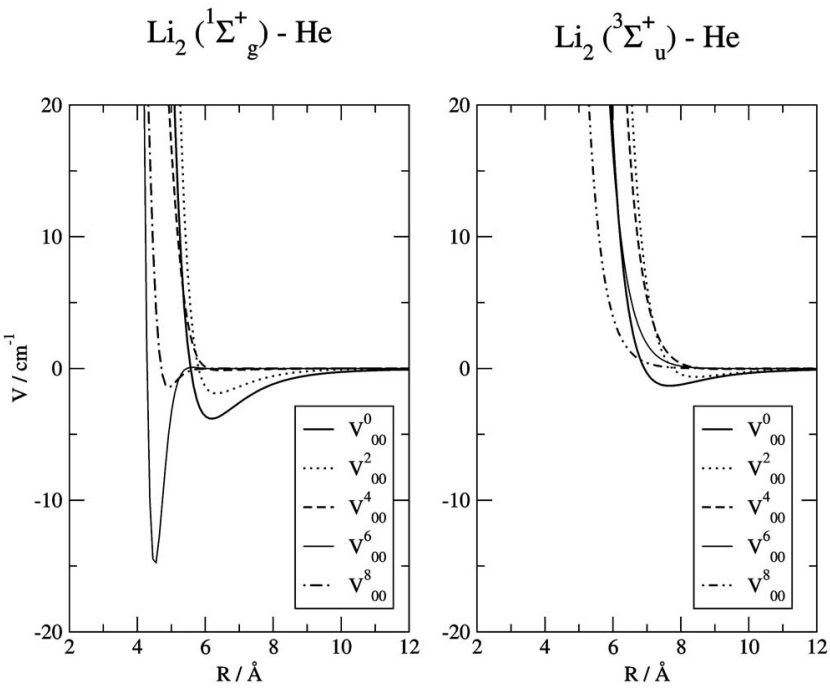

FIG. 4. Computed coefficients of the multipolar expansion [Eq. (3)] $V_{v v^{\prime}}^{\lambda}$ for the singlet dimer state (left) and for the triplet state (right), averaged over the ground vibrational states of each target molecule.

values. Each of the final $\lambda$ terms in expansion (3), in fact, can be approximately related to that transition since we know that $\lambda \sim \Delta j{ }^{26}$ The two panels in Fig. 4 report a comparison between some of the coefficients computed for the singlet state (left) and those computed for the triplet state (right).

The interesting difference between the two panels is that in the case of the triplet, the coefficients associated with $\lambda$ $>0$ have, at ultralow energies, the onset of their repulsive regions radially located outside that of the coefficient of $\lambda$ $=0$. On the contrary, the singlet target state (left panel) exhibits only the $\lambda=2$ and 4 coefficients with such behavior. This suggests that in the case of the triplet state, the values of the integrals of Eq. (3) will be larger over the radial range sampled during collisions, leading to larger inelastic flux components; therefore, the molecular target will undergo, over the relevant trajectories, a stronger coupling between non-neighboring rotational levels, in analogy to what we have seen for the vibrational coupling potentials of Fig. 3. The collisions for rotational quenching will thus become more efficient than in the case of a singlet state since the triplet target will have more extended interaction regions outside the location of its spherical repulsive "wall" for $\lambda=0$, a feature that is missing in the interaction potential for the singlet dimer.

\section{RESULTS FROM QUANTUM DYNAMICS}

The quantum dynamics was treated within a timeindependent formulation using the familiar coupled-channel approach in a space-fixed reference frame and employing our modified variable phase integrator to handle the necessary long-range potential regions acting at ultralow energies. ${ }^{26,27}$ These equations ${ }^{28}$ are propagated up to the asymptotic region where the solution matrix $\Psi(R)$ is obtained and where the final computed scattering matrix $\mathbf{S}$ yields the corresponding state-to-state superelastic cross sections 


$$
\begin{aligned}
\sigma_{v j, v^{\prime} j^{\prime}}\left(k_{v j}^{2}\right)= & \frac{\pi}{(2 j+1) k_{v j}^{2}} \sum_{J}(2 J+1) \sum_{l, l^{\prime}} \\
& \times\left|\delta_{l v j, l^{\prime} v^{\prime} j^{\prime}}-S_{v j, v^{\prime} j^{\prime}}\right|^{2},
\end{aligned}
$$

and the summed quenching cross sections

$$
\sigma_{v j}=\sum_{v^{\prime}<v} \sum_{j^{\prime}} \sigma_{v j, v^{\prime} j^{\prime}}
$$

At vanishing collision energies, we can then expand the elastic $\mathbf{S}$ matrix elements in the power of $k,{ }^{29}$

$$
S_{v j, v j} \simeq 1+2 i \delta_{v j}(k)=1-2 i k\left(\alpha_{v j}-i \beta_{v j}\right)=1-2 i k a_{v j},
$$

which allows us to define the complex scattering length $a_{v j}$ for the $|v j\rangle$ channel and its real, $\alpha_{v j}$, and imaginary, $\beta_{v j}$, parts. They are also related to the elastic and inelastic components of the partial integral cross sections at vanishing energy,

$$
\begin{aligned}
& \sigma_{v j}^{\mathrm{el}}=4 \pi\left|a_{v j}\right|^{2} \quad \text { as } k_{v j} \rightarrow 0, \\
& \sigma_{v j}^{\text {inel }}=4 \pi \beta_{v j} / k_{v j} \quad \text { as } k_{v j} \rightarrow 0 .
\end{aligned}
$$

We can further obtain the quenching rates down to all open channels for any selected initial state $|v j\rangle,{ }^{29}$

$$
R_{v j}=\frac{4 \pi \hbar}{\mu} \beta_{v j}
$$

The size and sign of the complex scattering length for each initial channel $|v j\rangle$ gives us information on the behavior of the bound states or resonances close to the dissociation limit of the complex; ${ }^{29}$ i.e., we can tell about the existence of zero-energy resonances, of virtual states, or of bound states of the complex near the dissociation limit by the sign of the real part of the corresponding scattering length. ${ }^{30}$ If the scattering is inelastic as in this case, these energies are complex and their imaginary parts therefore gives us additional information on the lifetimes of the metastable states. ${ }^{29,31,32}$

The radial coupled equations have been integrated out to $R_{\max }=1500 \AA$ and down to $10^{-6} \mathrm{~cm}^{-1}$ in terms of collision energies. The number of total angular momenta needed to obtain converged cross sections was $J_{\text {tot }}=0,1$, and 2 only, with the $s$-wave contributions dominating the scattering below $10^{-3} \mathrm{~cm}^{-1}$. The number of coupled rotational channels within each vibrational manifold was up to $j_{\max }=18$ for all vibrational states examined, and $B_{\text {rot }}$ was modified depending on the specific vibrational state. The coupled vibrational channels were always given by all the lower open states of the asymptotic triplet target plus two closed vibrational channels above each initial threshold. The details of the corresponding calculations of the singlet target state were already given in Ref. 14.

The calculations reported by the right panel in Fig. 5 show the elastic cross sections for different initial states of the triplet case computed here: For comparison, we also show in the left panel some of the earlier results ${ }^{13}$ for the $\mathrm{Li}_{2}$

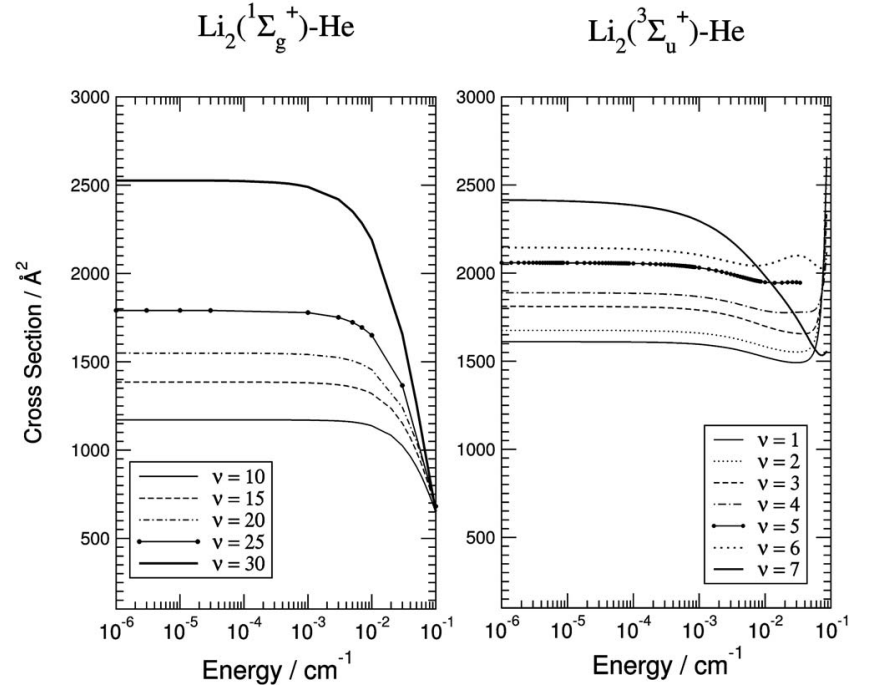

FIG. 5. Computed state-dependent elastic cross sections down to Wigner's regime of Ref. (13). The left panel refers to the singlet target state calculations, while the right panel is for the triplet state.

singlet state. All cross section calculations have been converged numerically from the lowest to the highest energies considered in the present work.

It is interesting to note how the elastic cross sections depend on energy from about $10^{-3} \mathrm{~cm}^{-1}$ down to our lowest collision energies: Both systems clearly appear to have reached Wigner's regime ${ }^{33}$ where the scattering is dominated by $s$-wave contributions. Furthermore, we see that the elastic cross sections for the triplet target state are all larger than the ones for the singlet, where only transitions from much higher vibrational levels (e.g., the solid line corresponding to $v_{i}$ $=30$ ) get reasonably close in size to those for the triplet (see Ref. 13).

Such differences are further visible when looking at the data relative to the actual rovibrational quenching processes, reported in Fig. 6, where the two systems are again compared in the two panels. The computed quenching cross sec-

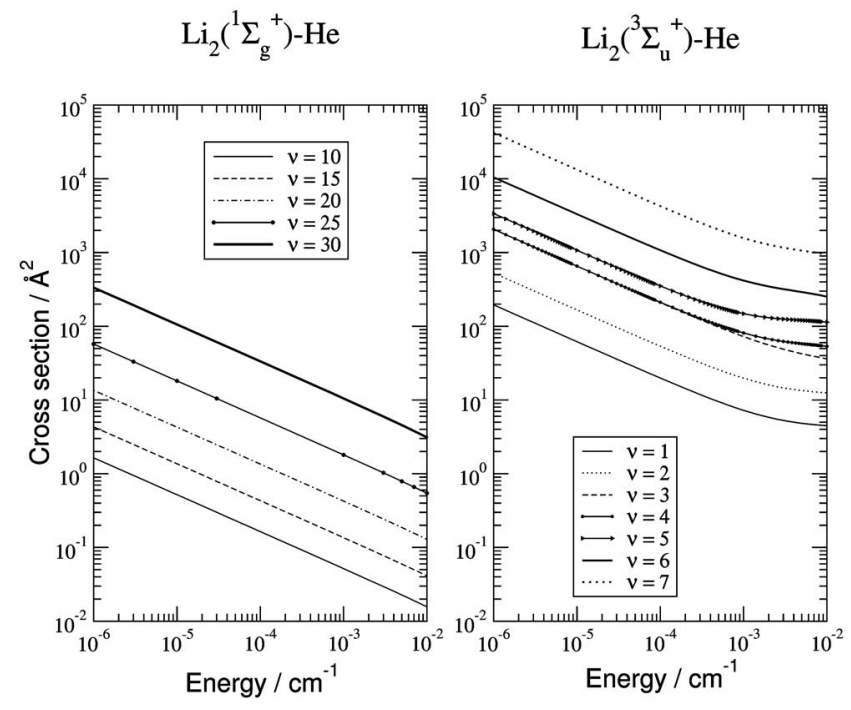

FIG. 6. Computed quenching cross sections for different initial vibrational states of the target states. Singlet data: left panel; triplet data: right panel. 

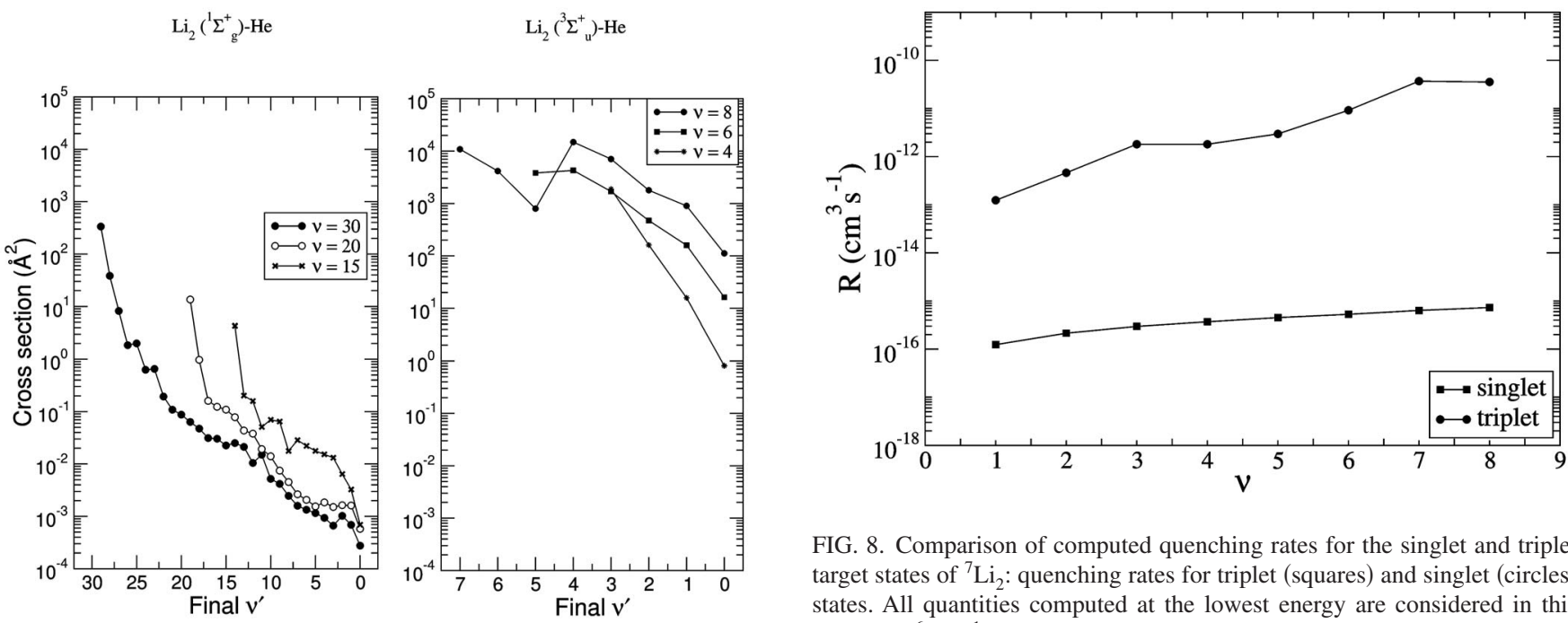

FIG. 8. Comparison of computed quenching rates for the singlet and triplet target states of ${ }^{7} \mathrm{Li}_{2}$ : quenching rates for triplet (squares) and singlet (circles) states. All quantities computed at the lowest energy are considered in this work $\left(10^{-6} \mathrm{~cm}^{-1}\right)$.

FIG. 7. Computed state-to-state vibrational quenching cross sections for the singlet state target (left panel) and the triplet state (right panel). See text for details. All quantities computed at the lowest energy are considered in this work $\left(10^{-6} \mathrm{~cm}^{-1}\right)$.

tions from Eq. (6) are obtained from $|v, j=0\rangle$ initial internal states and are therefore summed over all the many energetically open final states of each target.

The quenching cross sections are larger than those computed for the singlet: The features of the potential associated with the ${ }^{3} \Sigma_{u}^{+}$target discussed earlier are responsible for this behavior and for the increased quenching efficiency of this spin state of $\mathrm{Li}_{2}$. The cross sections presented in Fig. 7 further show the state-to-state cross sections: They correspond to $|j=0\rangle$ as the initial rotational state and are summed over all final rotational levels within each final vibrational state $\sum_{j^{\prime} \neq 0} \sigma\left(v, 0 \rightarrow v^{\prime} j^{\prime}\right)$. The results confirm the marked increase in efficiency of the quenching process when the triplet state of ${ }^{7} \mathrm{Li}_{2}$ is considered: All its state-to-state cross sections are several orders of magnitude larger than those exhibited by the singlet.

A summary of the computed scattering observables in the case of the triplet target is given in Table I, while in Fig. 8 , we compare the computed rates for the quenching processes in both spin states. All calculations refer to the lowest collision energy we have considered here.

The results for the scattering length (real part) indicate that in the triplet interaction with ${ }^{4} \mathrm{He}$, that quantity always

remains positive, and not very large, as the initial $|v\rangle$ state is changed. Such a result indicates, therefore, that the interaction potential is always capable of supporting a true bound state near the top of its well and that such a state appears to be located within $50 \mathrm{~cm}^{-1}$ from the potential rim. Thus, the possibility shown earlier by the singlet interaction to generate virtual states at ultralow energies [which we had discussed in our work $\left.{ }^{13}\right]$ becomes here the ability of the triplet potential to form additional bound states near the threshold.

The comparison between quenching rates (Fig. 8) further shows marked changes since the rovibrational quenching rates for the triplet are nearly three orders of magnitude larger than those for the singlet target. In other words, spinstretched dimer molecules of ${ }^{7} \mathrm{Li}$ are found by our calculations to be much more efficient in undergoing collisional quenching by $\mathrm{He}$ atoms than the singlet spin state. Although the possibility always exists that improving calculations for the relevant PES would be likely to also alter the size of the cross sections, we believe that the relative trend discussed here will not be significantly modified, especially when considering the optimized features of the present PESs as we discussed earlier in this paper.

\section{PRESENT CONCLUSIONS}

In this paper, we have analyzed in some detail the quantum dynamics associated with the rovibrational quenching at

TABLE I. Computed scattering length values, real and imaginary parts $\alpha$ and $\beta$, metastable bound state energies $E\left(\mathrm{~cm}^{-1}\right)$ and their lifetimes $\Gamma$ (ns), and total relaxation rates at vanishing temperatures for the triplet target state $R_{v}^{T}$ (in units of $\mathrm{cm}^{3} \mathrm{~s}^{-1}$ ).

\begin{tabular}{lccccc}
\hline \hline$\nu$ & $\alpha(\AA)$ & $\beta(\AA)$ & $E\left(\mathrm{~cm}^{-1}\right)$ & $\Gamma(\mathrm{ns})$ & $R_{\nu}^{T}(T \rightarrow 0)$ \\
\hline 1 & 11.32 & $6.66 \cdot 10^{-3}$ & $-4.22 \cdot 10^{-2}$ & 53.0 & $1.71 \cdot 10^{-13}$ \\
2 & 11.54 & $17.9 \cdot 10^{-3}$ & $-4.07 \cdot 10^{-2}$ & 20.9 & $4.61 \cdot 10^{-13}$ \\
3 & 12.00 & $70.7 \cdot 10^{-3}$ & $-3.76 \cdot 10^{-2}$ & 5.96 & $1.81 \cdot 10^{-12}$ \\
4 & 12.25 & $70.5 \cdot 10^{-3}$ & $-3.61 \cdot 10^{-2}$ & 6.37 & $1.81 \cdot 10^{-12}$ \\
5 & 12.80 & $11.5 \cdot 10^{-2}$ & $-3.31 \cdot 10^{-2}$ & 4.44 & $2.96 \cdot 10^{-12}$ \\
6 & 13.06 & $35.7 \cdot 10^{-2}$ & $-3.17 \cdot 10^{-2}$ & 1.52 & $9.19 \cdot 10^{-12}$ \\
7 & 13.78 & $14.3 \cdot 10^{-1}$ & $-2.76 \cdot 10^{-2}$ & 0.45 & $3.69 \cdot 10^{-11}$ \\
8 & 12.00 & $13.8 \cdot 10^{-1}$ & $-3.62 \cdot 10^{-2}$ & 0.31 & $3.55 \cdot 10^{-11}$ \\
\hline \hline
\end{tabular}


ultralow energies of the spin-stretched electronic state of the neutral lithium dimer, $a^{3} \Sigma_{u}^{+}$, in collision with ${ }^{4} \mathrm{He}$ as a buffer gas. The interest is related to obtaining information, for now mainly from a computational analysis, on the changes in the efficiency, with respect to their closed-shell counterparts, of the quenching of the internal degrees of freedom in simple molecules formed in their spin-stretched states.

The general features of the newly computed vibrational dependence of the potential for the triplet target interacting with He indicate a slightly stronger dynamical couplings between the vibrational levels of the triplet dimer with respect to those for the singlet. Furthermore, we have shown that the anisotropic coupling responsible for driving rotational excitations during the collisions is stronger in the case of the triplet than for the singlet. Hence, the differences in interaction forces between the two systems translate into marked differences in the collisional quenching efficiency of rovibrational states at ultralow energies.

As far as we are aware of, this is the only computational comparison of quenching efficiencies and specific quenching rates for an important dimer molecule, ${ }^{7} \mathrm{Li}_{2}$, considered in two different spin states and interacting with a common buffer-gas partner such as ${ }^{4} \mathrm{He}$. One of the clear results from the present calculations is that spin-stretched states, despite exhibiting similarly weak interaction PESs, show marked differences in the dynamical coupling terms, which distribute collisional flux into the different rovibrational final channels. In particular, the present case indicates a marked increase in quenching efficiency with respect to the singlet state when one moves from the $X^{1} \Sigma_{g}^{+}$to the $a^{3} \Sigma_{u}^{+}$state of the lithium dimer.

\section{ACKNOWLEDGMENTS}

The financial support of the Research Committee of the University of Rome "La Sapienza," of the PRIN funds of the Ministry for University and Research, of the CASPUR Supercomputing Consortium, and of the INTAS project on "Atomic and Molecular Clusters" is gratefully acknowledged.

\footnotetext{
${ }^{1}$ For example, see J. L. Roberts, N. R. Claussen, S. T. Thompson, and C. E. Wieman, Phys. Rev. Lett. 86, 4211 (2001).

${ }^{2}$ J. Herbig, T. Kraemer, M. Mark, T. Weber, C. Chin, H. C. Naegerl, and
}

R. Grimm, Science 301, 1510 (2003).

${ }^{3}$ S. Dürr, T. Volz, A. Marte, and G. Rempe, Phys. Rev. Lett. 92, 020406 (2004).

${ }^{4}$ C. A. Regal, C. Ticknor, J. L. Bohn, and D. S. Jin, Nature (London) 424, 47 (2003)

${ }^{5}$ K. E. Strecker, G. B. Partridge, and R. G. Hulet, Phys. Rev. Lett. 91, 080406 (2004).

${ }^{6}$ C. A. Regal, M. Greiner, and D. S. Jin, Phys. Rev. Lett. 92, 040403 (2004).

${ }^{7}$ M. W. Zwierlein, C. A. Stan, C. H. Schunck, S. M. F. Rampach, A. J. Kerman, and W. Ketterle, Phys. Rev. Lett. 92, 120403 (2004).

${ }^{8}$ J. D. Weinstein, R. de Cavalho, T. Guillet, B. Friederich, and J. Doyle, Nature (London) 395, 148 (1998).

${ }^{9}$ H. L. Bethlem and G. Meijer, Int. Rev. Phys. Chem. 22, 73 (2003).

${ }^{10}$ H. W. Chan, A. T. Black, and V. Vuletic, Phys. Rev. Lett. 90, 063003 (2003).

${ }^{11}$ T. Köhler, K. Goral, and P. S. Julienne, Rev. Mod. Phys. 78, 1311 (2006).

${ }^{12}$ R. V. Krems, Int. Rev. Phys. Chem. 24, 99 (2005).

${ }^{13}$ E. Bodo, F. A. Gianturco, and E. Yurtsever, Phys. Rev. A 73, 052715 (2006).

${ }^{14}$ E. Bodo and F. A. Gianturco, Europhys. Lett. 77, 33001 (2007).

${ }^{15}$ J. J. Gilijamse, S. Hoekstra, S. Y. T. van de Meerakker, G. C. Groenenboom, and G. Meijer, Science 313, 1617 (2007).

${ }^{16}$ S. Willitsch, M. T. Bell, A. D. Gingell, S. R. Procter, and T. P. Softley, Phys. Rev. Lett. 100, 043203 (2008).

${ }^{17}$ E. Bodo, F. A. Gianturco, E. Yurtsever, and M. Yurtsever, Mol. Phys. 103, 3223 (2005)

${ }^{18}$ M. Wernli, E. Bodo, and F. A. Gianturco, Eur. Phys. J. D 45, 267 (2007).

${ }^{19}$ E. Bodo, F. A. Gianturco, and E. Yurtsever, J. Low Temp. Phys. 138, 259 (2005).

${ }^{20}$ A. Aguado, C. Tablero, and M. Paniagua, Comput. Phys. Commun. 1087, 259 (1998)

${ }^{21}$ K. T. T. U. Kleinekathofer, J. P. Toennies, and C. L. Yiu, Chem. Phys. Lett. 249, 257 (1996).

${ }^{22}$ M. D. Halls, H. B. Schlegel, M. J. De Witt, and G. W. F. Drake, Chem. Phys. Lett. 339, 427 (2001).

${ }^{23}$ R. Cotè, A. Dalgarno, and J. Jamieson, Phys. Rev. A 50, 399 (1994).

${ }^{24}$ J. M. Hutson and P. Soldán, Int. Rev. Phys. Chem. 25, 497 (2006).

${ }^{25}$ C. Linton, T. L. Murphy, F. Martin, R. Bacis, and J. Verges, J. Chem. Phys. 91, 6036 (1989).

${ }^{26}$ For example, see A. M. Arthurs and A. Dalgarno, Proc. R. Soc. London, Ser. A 256, 540 (1960).

${ }^{27}$ W. Eastes and D. Secrest, J. Chem. Phys. 56, 640 (1972).

${ }^{28}$ R. Martinazzo, E. Bodo, and F. A. Gianturco, Comput. Phys. Commun. 151, 187 (2003).

${ }^{29}$ N. Balakrishnan, R. Forrey, and A. Dalgarno, Chem. Phys. Lett. 280, 1 (1997).

${ }^{30}$ For example, see C. J. Joachain, Quantum Collision Theory (NorthHolland, Amsterdam, 1975)

${ }^{31}$ For a recent analysis, see J. M. Hutson, New J. Phys. 9, 152 (2007).

${ }^{32}$ For example, see E. Bodo and F. A. Gianturco, Int. Rev. Phys. Chem. 25, 283 (2006).

${ }^{33}$ P. E. Wigner, Phys. Rev. 73, 1002 (1948). 